\title{
INTERPRETAÇÃO DE DADOS OBTIDOS EM TESTES DE VIGOR PARAA COMPARAÇÃO QUALITATIVA ENTRE LOTES DE SEMENTES DE MILHO ${ }^{1}$
}

\author{
LEILAMARTINS², WALTERRODRIGUESDASILVA ${ }^{3}$
}

\begin{abstract}
RESUMO - O objetivo desta pesquisa foi estudar, em lotes de sementes de milho, a possibilidade de estabelecer relações entre os dados de vigor obtidos em laboratório com os verificados no campo. Para tanto, foram utilizados 28 lotes (tratamentos) de sementes de milho (cv. Al Bandeirante) padronizadas em relação ao grau de umidade, à largura, ao comprimento e à germinação. Posteriormente, foram conduzidos testes de vigor em laboratório (conjunto A: frio sem solo, envelhecimento acelerado, germinação sob estresse hídrico e comprimentos da raiz, da parte aérea e da plântula com variações na disponibilidade hídrica) e em campo (conjunto B: emergência das plântulas). Para a comparação entre as médias, foi utilizado o teste de Tukey a 5\% de probabilidade; complementarmente, os lotes receberam pontuações obedecendo a ordenação hierárquica de desempenho verificada em cada avaliação, segundo critérios de classificações estatística e absoluta, para serem agrupados de acordo com o desempenho verificado nos conjuntos (A e B) de testes. A interpretação de dados de vigor, oriundos de um conjunto de testes, adquire complexidade diretamente proporcional aos números de testes e de lotes colocados em comparação. Nessa situação, a classificação absoluta supera a classificação estatística nas tarefas de hierarquizar qualitativamente lotes de sementes de milho, segundo o desempenho geral, e de quantificar o relacionamento entre as respostas provenientes dos testes de laboratório com as obtidas em campo.
\end{abstract}

Termos para indexação: Zea mays, germinação, avaliação da qualidade.

\section{INTERPRETATION OF DATA OBTAINED IN VIGOR TESTS FOR QUALITY COMPARISON AMONGCORN SEEDLOTS}

\begin{abstract}
The objective of this research was to study, using corn seed lots, the possibility of establishing relations between vigor data obtained in the laboratory with those observed in the field. To do so, twenty eight corn seed lots (treatments) of the Al Bandeirante open-pollinated variety were standardized regarding seed moisture content, width, length and germination. Afterwards, seed vigor tests were conducted in the laboratory (group A: modified cool germination test; accelerated aging; germination under variations in water availability; radicle, shoot and seedling length under variations in water availability) and in the field (group B: seedling emergence). In order to compare means the 5\% Tukey test was used; in addition, the seed lots were assigned points according to a ranking order of performance observed in each evaluation, following criteria of absolute and statistical classifications, so to group them in order of performance in the A and B test groups. Interpretation of vigor data resulting from a group of tests acquires a complexity directly proportional to the number of tests and lots being compared. In this way, the use of absolute classification surpasses the statistical classification in the tasks of qualitatively ranking corn seed lots according to their general performance and, in addition, quantifies the relationship among the responses observed in the laboratory with those obtained in the field.
\end{abstract}

Index terms: Zea mays, germination, quality evaluation

\footnotetext{
${ }^{1}$ Submetido em 01/06/2004. Aceito para publicação em 08/02/2005. Apoiado financeiramente pela Fundação de Amparo à Pesquisa do Estado de São Paulo (FAPESP).
}

${ }^{2}$ Laboratório Central de Sementes e Mudas, DSMM - CATI. Caixa Postal
1291, CEP 13073-001 Campinas (SP), Brasil / E-mail: leila@cati.sp.gov.br ${ }^{3}$ Bolsista do CNPq, Departamento de Produção Vegetal, ESALQ/USP.Caixa Postal 09, CEP 13418-900 Piracicaba (SP), Brasil / E-mail: wrsilva@carpa.ciagri.usp.br 


\section{INTRODUÇÃO}

A constatação de limitações, para estimar de modo abrangente o potencial fisiológico das sementes, estimulou a concepção de determinações, denominadas testes de vigor, encarregadas de avaliar aspectos qualitativos não detectados pelo teste de germinação (Vieira et al., 1994; Carvalho \& Nakagawa, 2000).

Entre os testes de vigor desenvolvidos, destacam-se os voltados à estimativa do comportamento fisiológico das sementes em situações ambientais desfavoráveis (ISTA, 1981; AOSA, 1983; Marcos Filho et al., 1987; Carvalho \& Nakagawa, 2000) como, por exemplo, as presentes nos testes de frio, de envelhecimento acelerado e de estresse hídrico. Porém, admitindo que um teste, isoladamente, seja incapaz de antever o desempenho fisiológico nas diversas condições ambientais possíveis, o emprego simultâneo de várias determinações tem sido preconizado para a validação das estimativas de vigor (Hampton \& Coolbear, 1990; Marcos Filho, 1998).

Dependendo dos testes utilizados, as informações obtidas podem ser distintas entre si (Grabe, 1976; Marcos Filho et al., 1984) e, em conseqüência, as comparações, entre lotes ou tratamentos, ficam prejudicadas na medida em que os desempenhos relativos variam segundo o teste aplicado. Essa ocorrência dificulta a definição de ações em alguns programas de controle de qualidade que, como alternativa, tem adotado reduzido número de testes para embasar o seu processo decisório.

Dessa maneira, o estabelecimento de procedimentos capazes de fornecer respostas, a partir da variabilidade existente nas informações provenientes de um conjunto de testes de vigor, constitui-se em demanda a ser pesquisada. Caliari \& Silva (2001), trabalhando com o tema, aplicaram critérios de interpretação que, utilizando comparações em bases estatísticas e não estatísticas, permitiram classificações para o desempenho geral de lotes submetidos a vários testes.

Assim, a presente pesquisa objetivou estudar, em lotes de sementes de milho, a possibilidade de estabelecer relações entre os dados de vigor obtidos em laboratório com os verificados em campo.

\section{MATERIAL E MÉTODOS}

As sementes de milho (cv. Al Bandeirante) utilizadas, representando 28 lotes (tratamentos) com grau de umidade (Brasil, 1992) de 13,3 $\pm 0,9 \%$, foram padronizadas em relação à largura (20/ 64") e ao comprimento (longas) e, em seguida, submetidas à avaliação da germinação segundo os procedimentos descritos nas Regras para Análise de Sementes (Brasil, 1992). Posteriormente, foram conduzidos conjuntos de testes de vigor, em laboratório e em campo, empregando os procedimentos descritos a seguir.

Conjunto A (dados obtidos em laboratório):

a) Frio sem solo: foi conduzido com 50 sementes por repetição que, previamente submetidas a $10^{\circ} \mathrm{C}$ por sete dias em rolo de papel umedecido com volume $(\mathrm{mL})$ de água equivalente a 2,5 vezes a sua massa $(\mathrm{g})$ sem hidratação, foram mantidas em germinador $\left(30^{\circ} \mathrm{C}\right)$ por sete dias para a avaliação da porcentagem de plântulas normais (Marcos Filho et al., 1987).

b) Envelhecimento acelerado: foram utilizadas 50 sementes por repetição distribuídas sobre bandeja de tela de alumínio fixada no interior de caixa plástica (mini-câmara), contendo $40 \mathrm{~mL}$ de água, mantida a $42^{\circ} \mathrm{C}$ por 96 horas (AOSA, 1983). Decorrido este período, as sementes foram colocadas para germinar seguindo a metodologia utilizada no teste de germinação (Brasil, 1992). A avaliação das plântulas, realizada no quarto dia após a semeadura, considerou a porcentagem de plântulas normais conforme o descrito por Marcos Filho et al. (1987).

c) Germinação sob estresse hídrico: foi conduzida em substratos com potenciais hídricos (-200 e -300 kPa) obtidos, através do uso de soluções aquosas contendo polietileno glicol (PEG 6000), segundo os cálculos de Villela et al. (1991). O papel substrato, umedecido com as soluções em quantidade (mL) equivalente a 2,5 vezes a sua massa ( $g$ ) sem hidratação, foi semeado, enrolado e embalado em saco plástico para posterior colocação em germinador a $30^{\circ} \mathrm{C}$. Foram utilizadas 50 sementes por repetição e seguidos os procedimentos de avaliação descritos para o teste de germinação.

d) Comprimentos da raiz, da parte aérea e da plântula com variações na disponibilidade hídrica: em substratos com 0, -200 e -300 $\mathrm{kPa}$, preparados da mesma forma adotada nos testes de germinação, 10 sementes por repetição foram instaladas eqüidistantemente sobre o terço superior do papel substrato. $\mathrm{O}$ substrato, enrolado no sentido do comprimento, foi embalado em saco plástico e levado para germinador a $30^{\circ} \mathrm{C}$, na ausência de luz, de maneira que as radículas ficassem voltadas para baixo. No final do período de quatro dias foram tomadas, em todos os indivíduos, as distâncias ( $\mathrm{mm}$ ) do ápice da raiz ao nó cotiledonar (comprimento da raiz) e deste ao ápice do epicótilo (comprimento da parte aérea); o somatório de ambas as medidas representou o comprimento da plântula. Foi calculado, para cada determinação, o quociente entre o somatório dos comprimentos verificados e o número total de indivíduos instalados.

\section{Conjunto B (dados obtidos em campo)}

a) Emergência das plântulas: foi realizada com 50 sementes por repetição, em 4 épocas (setembro, outubro, novembro e dezembro) e 5 locais (blocos) situados nos municípios de Aguaí/ SP, Ibitinga/SP, Mandurí/SP, Marília/SP e Paraguaçu Paulista/SP. A semeadura foi realizada a $3,0 \mathrm{~cm}$ de profundidade, em linhas com $10,0 \mathrm{~m}$ de comprimento e distanciadas $0,5 \mathrm{~m}$ entre si, em solo 
suficientemente úmido para permitir a hidratação inicial das sementes. As contagens, efetuadas no 15ำ dia após a semeadura, foram utilizadas para determinar a porcentagem de plântulas emersas conforme os procedimentos relatados por Marcos Filho et al. (1987).

Nas determinações realizadas em laboratório (conjunto A), foram empregadas 4 repetições em delineamento inteiramente casualizado; as determinações realizadas em campo (conjunto B), durante cada época experimental, foram conduzidas em blocos (5) casualizados contendo, cada um, sub-blocos (4) com 4 repetições. Para a comparação entre as médias, foi utilizado o teste de Tukey a 5\% de probabilidade.

Adicionalmente, conforme o exemplificado nas Tabelas 1 e 2, os lotes receberam pontuações segundo a ordenação hierárquica de desempenho verificada em cada avaliação. Para tanto, foram consideradas classificações, semelhantes às empregadas por Caliari \& Silva (2001), baseadas nas diferenças indicadas pelo teste de Tukey (classificação estatística) e pelos valores absolutos (classificação absoluta).

$\mathrm{Na}$ classificação estatística, em cada teste, foi atribuída a cada um dos lotes a pontuação resultante do somatório das pontuações positivas ou nulas (número de lotes estatisticamente inferiores) e negativas ou nulas (número de lotes estatisticamente superiores). Na classificação absoluta, a pontuação foi representada pelo número (nulo ou positivo) de lotes superados em valor absoluto, independentemente das indicações estatísticas. Em ambas as classificações, a soma dos valores obtidos em todos os testes constituiu a pontuação geral do lote em cada um dos conjuntos (A e B) de determinações.

TABELA 1. Classificação estatística: exemplo hipotético de pontuação por teste, de pontuação geral, de formação de grupos SM (Superior ao Médio), IM (Inferior ao Médio) e M (Médio = pontuação nula) nos conjuntos de testes A (realizados em laboratório) e B (realizados em campo), de contagem do número de lotes pertencentes ao mesmo grupo em ambos os conjuntos de testes ( $\mathrm{A}$ e B) e de cálculo de taxa de coincidência ( $\mathrm{TC}=$ número de lotes pertencentes ao mesmo grupo.número total de lotes -1.100).

\begin{tabular}{|c|c|c|c|c|c|c|c|c|c|c|c|c|}
\hline \multicolumn{13}{|c|}{ Classificação estatística } \\
\hline \multicolumn{6}{|c|}{ Conjunto A (testes realizados em laboratório) } & \multicolumn{6}{|c|}{ Conjunto B (testes realizados em campo) } & \multirow{3}{*}{$\begin{array}{c}\text { Conjuntos A e B } \\
\text { Presenças no } \\
\text { mesmo grupo }\end{array}$} \\
\hline \multirow[b]{2}{*}{ Lotes } & \multicolumn{2}{|c|}{ Teste 1} & \multirow{2}{*}{$\frac{\text { Teste } 2}{\text { Dados Pontuação }}$} & \multicolumn{2}{|c|}{ PontuaçãoGrupo } & \multirow{2}{*}{\multicolumn{2}{|c|}{$\frac{\text { Teste } 3}{\text { Dados Pontuação }}$}} & \multirow{2}{*}{\multicolumn{2}{|c|}{$\frac{\text { Teste } 4}{\text { Dados Pontuação }}$}} & \multicolumn{2}{|c|}{$\overline{\text { PontuaçãoGrupo }}$} & \\
\hline & Dados & Pontuação & & Geral & $\mathrm{M}=0$ & & & & & Geral & $\mathrm{M}=0$ & \\
\hline L1 & $100 \mathrm{a}$ & $2+0=2$ & 98 b $1+(-1)=0$ & 2 & SM & 96 a & $2+0=2$ & $90 \mathrm{bc}$ & $+(-2)=-2$ & 0 & M & 0 \\
\hline L2 & $99 \mathrm{ab}$ & $1+0=1$ & $97 \mathrm{bc} 0+(-1)=-1$ & 0 & M & $86 \mathrm{c}$ & $0+(-4)=-4$ & $97 \mathrm{a}$ & $2+0=2$ & -2 & IM & 0 \\
\hline L3 & $90 \mathrm{abc}$ & $0+0=0$ & $3+0=3$ & 3 & SM & $94 \mathrm{a}$ & $2+0=2$ & $95 \mathrm{a}$ & $2+0=2$ & 4 & SM & 1 \\
\hline L4 & 89 bc & $0+(-1)=-1$ & 96 c $0+(-3)=-3$ & -4 & IM & $90 \mathrm{~b}$ & $1+(-2)=-1$ & $92 \mathrm{ab}$ & $1+0=1$ & 0 & M & 0 \\
\hline L5 & $87 \mathrm{c}$ & $0+(-2)=-2$ & $1+0=1$ & -1 & IM & $92 \mathrm{ab}$ & $1+(0)=1$ & $86 \mathrm{c}$ & $0+(-3)=-3$ & -2 & IM & 1 \\
\hline
\end{tabular}

$$
\mathrm{TC}=2.5^{-1} \cdot 100=40 \%
$$

TABELA 2 - Classificação absoluta: exemplo hipotético de pontuação por teste, de pontuação geral, de formação de grupos SM (Superior ao Médio), IM (Inferior ao Médio) e M(Médio=somatório da maior e da menor pontuações. 2-1) nos conjuntos de testes A (realizados em laboratório) e B (realizados em campo), de contagem do número de lotes pertencentes no mesmo grupo em ambos os conjuntos de testes (A e B) e de cálculo da taxa de coincidência (TC=número de lotes presentes no mesmo grupo.número total de lotes -1.100$)$.

\begin{tabular}{|c|c|c|c|c|c|c|c|c|c|c|c|c|c|}
\hline \multicolumn{14}{|c|}{ Classificação absoluta } \\
\hline \multicolumn{7}{|c|}{ Conjunto A (testes realizados em laboratório) } & \multicolumn{6}{|c|}{ Conjunto B (testes realizados em campo) } & \multirow{3}{*}{$\begin{array}{c}\text { Conjuntos A e B } \\
\text { Presenças no } \\
\text { mesmo grupo }\end{array}$} \\
\hline & & Teste 1 & & Teste 2 & \multirow{2}{*}{$\begin{array}{l}\text { Pontuação } \\
\text { Geral }\end{array}$} & \multirow{2}{*}{$\begin{array}{c}\text { Grupo } \\
\mathrm{M}=(6+1) \cdot 2-1=3,5\end{array}$} & \multicolumn{2}{|r|}{ Teste 3} & \multicolumn{2}{|c|}{ Teste 4} & \multirow{2}{*}{$\begin{array}{c}\text { Pontuação } \\
\text { Geral }\end{array}$} & \multirow{2}{*}{$\begin{array}{c}\text { Grupo } \\
\mathrm{M}=(6+2) \cdot 2-1=4\end{array}$} & \\
\hline Lotes & $\overline{\text { Dados }}$ & s Pontuação & $\overline{\text { Dados }}$ & os Pontuação & & & $\overline{\text { Dados }}$ & os Pontuação & $\overline{\text { Dados }}$ & os Pontuação & & & \\
\hline L1 & 100 & 4 & 98 & 2 & 6 & SM & 96 & 4 & 90 & 1 & 5 & SM & 1 \\
\hline L2 & 99 & 3 & 97 & 1 & 4 & SM & 86 & 0 & 97 & 4 & 4 & M & 0 \\
\hline L3 & 90 & 2 & 100 & 4 & 6 & SM & 94 & 3 & 95 & 3 & 6 & SM & 1 \\
\hline L4 & 89 & 1 & 96 & 0 & 1 & IM & 90 & 1 & 92 & 2 & 3 & IM & 1 \\
\hline \multirow[t]{3}{*}{ L5 } & 87 & 0 & 99 & 3 & 3 & IM & 92 & 2 & 86 & 0 & 2 & IM & 1 \\
\hline & & & & & & & & & & & & & Total \\
\hline & & & & & & & & & & & & & $\mathrm{TC}=4.5^{-1} \cdot 100=80^{\circ}$ \\
\hline
\end{tabular}


Posteriormente, considerando a pontuação geral obtida pelos lotes em cada conjunto de testes, foi identificado o desempenho médio para a distribuição dos lotes em grupos superiores (SM), iguais (M) e inferiores (IM) ao desempenho médio, nas duas classificações. Na classificação estatística, o desempenho médio foi estabelecido na pontuação geral nula ( $n^{-}$de lotes estatisticamente superados $=\mathrm{n}^{\mathrm{o}}$ de lotes estatisticamente superiores); na classificação absoluta, o desempenho médio foi atribuído à média aritmética do somatório da maior e da menor pontuações gerais alcançadas pelos lotes.

Em seguida, obtidos os números de lotes presentes em grupos idênticos em ambos os conjuntos (A e B) de testes, foi calculada a taxa $(\%)$ de coincidência $\left(\mathrm{TC}=\mathrm{n}^{\mathrm{o}}\right.$ de lotes presentes no mesmo grupo $\mathrm{x} \mathrm{n}^{\mathrm{o}}$ total de lotes $^{-1} \times 10^{2}$ ) proporcionada, pelos critérios de pontuação, para relacionar os dados de laboratório (conjunto A) com os de campo (conjunto B).

\section{RESULTADOS E DISCUSSÃO}

Particularmente em relação à germinação, a ausência de distinção entre lotes constitui-se em justificativa para o uso dos testes de vigor (Marcos Filho et al., 1984; Carvalho \& Nakagawa, 2000) e, portanto, em premissa a ser atendida nos estudos direcionados à estimativa da habilidade dessas avaliações para hierarquizar o desempenho relativo de lotes ou de tratamentos submetidos à comparação. Os dados de germinação (Tabela 3), obtidos preliminarmente à condução dos demais testes, indicaram a existência de uniformidade qualitativa entre os lotes estudados.

Por sua vez, as estimativas de vigor demandam a utilização concomitante de diversos testes (Hampton \& Coolbear, 1990; Marcos Filho, 1998) em virtude da possibilidade do surgimento de divergências, entre as informações coletadas, originadas em particularidades inerentes à natureza de cada determinação (Grabe 1976; Marcos Filho et al. 1984). Como decorrência dessa diversidade nas respostas, a comparação entre os lotes adquire complexidade diretamente proporcional aos números de testes aplicados e de lotes a serem comparados. Sob esses aspectos, a observação dos dados coletados indica que, considerando as diferenciações estabelecidas pelo teste de Tukey (Tabelas 4 e 5), os testes de comprimento da raiz $(0 \mathrm{kPa})$, de comprimento da plântula $(0 \mathrm{kPa})$ e de emergência das plântulas (época 4$)$ foram os únicos a apresentar informações idênticas para a comparação entre os lotes; contudo, as respostas fornecidas, da mesma forma que o verificado para a germinação (Tabela 3 ), consideraram os lotes qualitativamente indiferenciáveis. Os demais testes apontaram, invariavelmente, diferenças qualitativas entre os lotes; no entanto, a ordenação relativa fornecida, segundo o desempenho dos lotes, foi particular para cada caso e, dessa maneira, ficou impossibilitada a definição de hierarquia que compatibilizasse as indicações provenientes do conjunto A (testes de laboratório) com as verificadas no conjunto B (testes de campo).

Diante dessa situação, foram aplicadas alternativas de interpretação baseadas nas classificações estatística e absoluta (Caliari \& Silva, 2001) que, por meio da atribuição de pontuações proporcionais ao vigor observado nos lotes em cada teste, originaram a formação de grupos (superior ao médio, médio e inferior ao médio) segundo o desempenho geral obtido dentro de cada conjunto (A e B) de testes.

Segundo os critérios empregados na classificação estatística, os lotes, perfazendo as pontuações gerais indicadas nas Tabelas 4 (conjunto A) e 5 (conjunto B), foram agrupados (Tabela 6) tomando como referência o desempenho médio representado pela pontuação nula (número de lotes superados = número de lotes superiores); calculada a taxa de coincidência, foi observado que, em $60,71 \%$ dos casos, os grupos atribuídos aos lotes foram idênticos nos conjuntos A e B.

Em relação à classificação absoluta, as pontuações gerais (Tabelas 7 e 8) observadas nos dois conjuntos, colocadas em comparação com o desempenho médio (média aritmética do somatório da maior e da menor pontuações alcançadas pelos lotes), permitiram a formação dos agrupamentos indicados na Tabela 9. Nessa situação, a taxa de coincidência, superando a verificada na classificação estatística, constatou similaridade em $75 \%$ dos casos.

TABELA 3. Dados da avaliação de germinação (G), em lotes de sementes de milho, obtidos anteriormente à realização das determinações do vigor em laboratório (conjunto A) e no campo (conjunto B) (1).

\begin{tabular}{ccccc}
\hline Lote $^{\text {o }}$ & $\mathrm{G}(\%)$ & & Lote $\mathrm{n}^{\mathbf{0}}$ & $\mathrm{G}(\%)$ \\
\cline { 1 - 2 } \cline { 5 - 5 } 2 & $86,2 \mathrm{a}$ & & 15 & $90,0 \mathrm{a}$ \\
2 & $88,7 \mathrm{a}$ & & 16 & $92,7 \mathrm{a}$ \\
3 & $89,4 \mathrm{a}$ & & 17 & $83,7 \mathrm{a}$ \\
4 & $91,2 \mathrm{a}$ & & 18 & $83,1 \mathrm{a}$ \\
5 & $82,1 \mathrm{a}$ & & 19 & $84,3 \mathrm{a}$ \\
6 & $91,7 \mathrm{a}$ & & 20 & $92,6 \mathrm{a}$ \\
7 & $91,7 \mathrm{a}$ & & 21 & $85,6 \mathrm{a}$ \\
8 & $87,0 \mathrm{a}$ & & 22 & $89,1 \mathrm{a}$ \\
9 & $92,9 \mathrm{a}$ & & 23 & $86,3 \mathrm{a}$ \\
10 & $91,3 \mathrm{a}$ & & 24 & $85,6 \mathrm{a}$ \\
11 & $87,3 \mathrm{a}$ & & 25 & $90,2 \mathrm{a}$ \\
12 & $90,8 \mathrm{a}$ & & 26 & $86,7 \mathrm{a}$ \\
13 & $84,5 \mathrm{a}$ & & 27 & $89,6 \mathrm{a}$ \\
14 & $86,5 \mathrm{a}$ & 28 & $83,5 \mathrm{a}$ \\
\hline
\end{tabular}

(1) Considerando todos os dados, médias seguidas de mesma letra não diferem entre si pelo Teste de Tukey a $5 \%$ de probabilidade. 


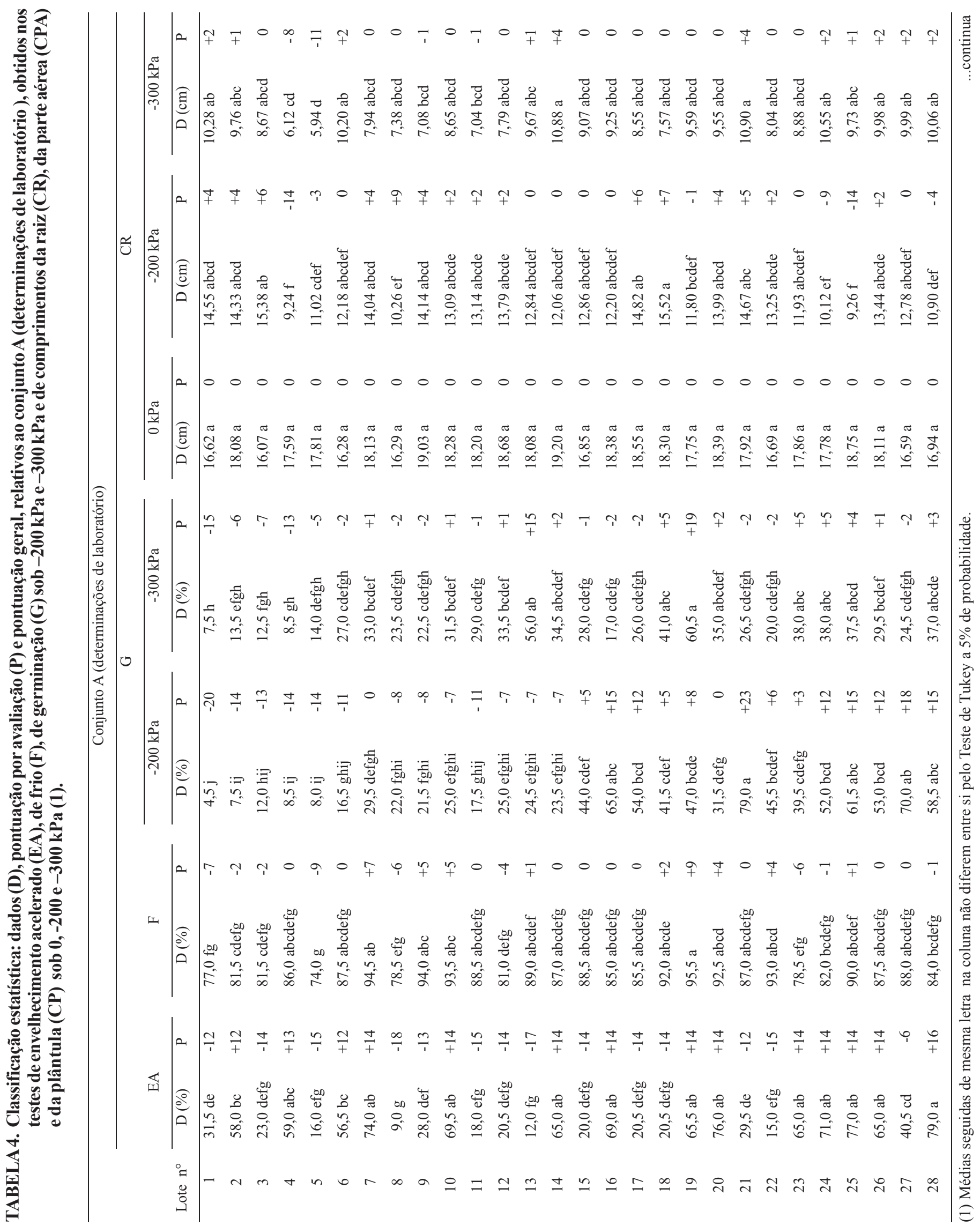




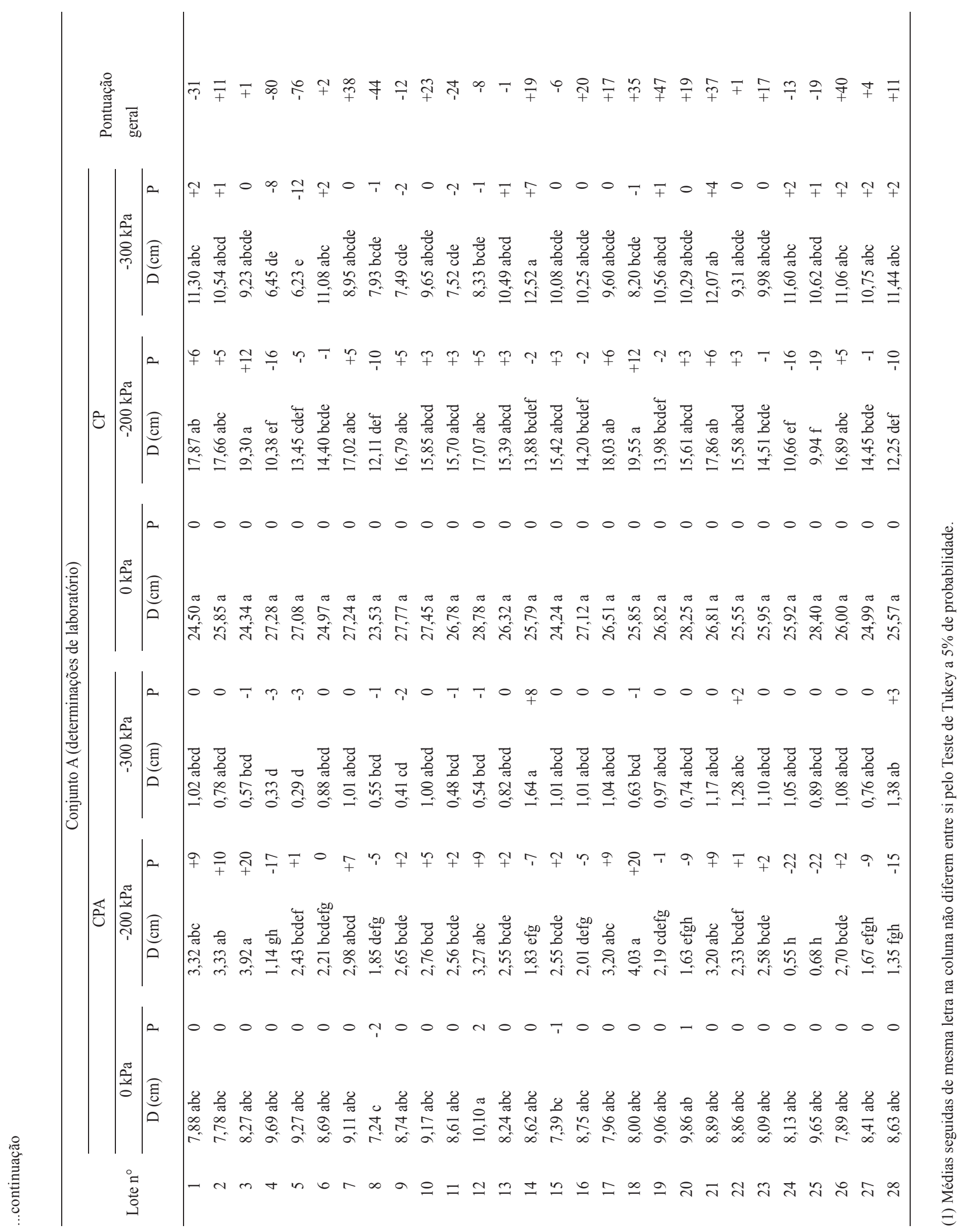


TABELA 5. Classificação estatística: dados (D), pontuação por avaliação (P) e pontuação geral, relativos ao conjunto $B$ (determinações de campo), obtidos nos testes de emergência das plântulas (EP) (1)

\begin{tabular}{|c|c|c|c|c|c|c|c|c|c|}
\hline \multirow{3}{*}{ Lote $\mathbf{n}^{\circ}$} & \multicolumn{8}{|c|}{ Conjunto B (determinações de campo) } & \multirow{3}{*}{ Pontuação geral } \\
\hline & \multicolumn{2}{|c|}{ EP (época 1) } & \multicolumn{2}{|c|}{ EP (época 2) } & \multicolumn{2}{|c|}{ EP (época 3) } & \multicolumn{2}{|c|}{ EP (época 4) } & \\
\hline & $\mathrm{D}(\%)$ & $\mathbf{P}$ & $\mathrm{D}(\%)$ & $\mathbf{P}$ & $\mathrm{D}(\%)$ & $\mathbf{P}$ & $\mathrm{D}(\%)$ & $\mathbf{P}$ & \\
\hline 1 & $87,88 \mathrm{ab}$ & +1 & $62,03 \mathrm{ab}$ & $\mathrm{O}$ & 86,64 abc & $\mathrm{O}$ & 89,19 a & $\mathrm{O}$ & +1 \\
\hline 2 & $86,17 \mathrm{ab}$ & +1 & $63,10 \mathrm{ab}$ & $\mathrm{O}$ & $85,83 \mathrm{abc}$ & $\mathrm{O}$ & $89,94 \mathrm{a}$ & $\mathrm{O}$ & +1 \\
\hline 3 & $82,50 \mathrm{abc}$ & O & $67,49 \mathrm{ab}$ & $\mathrm{O}$ & $86,68 \mathrm{abc}$ & $\mathrm{O}$ & $86,94 \mathrm{a}$ & $\mathrm{O}$ & O \\
\hline 4 & $87,80 \mathrm{ab}$ & +1 & $61,77 \mathrm{ab}$ & $\mathrm{O}$ & 85,37 abc & $\mathrm{O}$ & 86,75 a & $\mathrm{O}$ & +1 \\
\hline 5 & $73,30 \mathrm{c}$ & -22 & $51,48 \mathrm{~b}$ & -1 & $80,62 \mathrm{c}$ & -17 & $83,24 \mathrm{a}$ & $\mathrm{O}$ & -40 \\
\hline 6 & $82,56 \mathrm{abc}$ & O & $61,60 \mathrm{ab}$ & O & 87,46 abc & O & $87,97 \mathrm{a}$ & $\mathrm{O}$ & O \\
\hline 7 & $88,15 \mathrm{ab}$ & +1 & $64,83 \mathrm{ab}$ & O & $89,70 \mathrm{ab}$ & +1 & 89,40 a & $\mathrm{O}$ & +2 \\
\hline 8 & $80,94 \mathrm{bc}$ & -1 & $64,06 \mathrm{ab}$ & O & $83,20 \mathrm{bc}$ & -5 & 83,92 a & O & -6 \\
\hline 9 & $87,82 \mathrm{ab}$ & +1 & $56,51 \mathrm{ab}$ & O & $89,15 \mathrm{ab}$ & +1 & $87,78 \mathrm{a}$ & $\mathrm{O}$ & +2 \\
\hline 10 & $85,97 \mathrm{ab}$ & +1 & $69,57 \mathrm{ab}$ & $\mathrm{O}$ & 88,35 abc & $\mathrm{O}$ & $89,50 \mathrm{a}$ & $\mathrm{O}$ & +1 \\
\hline 11 & $87,84 \mathrm{ab}$ & +1 & $70,22 \mathrm{ab}$ & O & $89,82 \mathrm{ab}$ & +1 & 90,48 a & $\mathrm{O}$ & +2 \\
\hline 12 & 83,82 abc & +1 & 73,28 a & +1 & 88,06 abc & O & $90,22 \mathrm{a}$ & $\mathrm{O}$ & +2 \\
\hline 13 & 83,82 abc & O & $63,33 \mathrm{ab}$ & O & 84,57 abc & O & $89,15 \mathrm{a}$ & $\mathrm{O}$ & O \\
\hline 14 & $85,62 \mathrm{ab}$ & +1 & $62,32 \mathrm{ab}$ & O & 86,78 abc & O & $87,54 \mathrm{a}$ & O & +1 \\
\hline 15 & 86,58 abc & O & $63,43 \mathrm{ab}$ & O & $88,80 \mathrm{ab}$ & +1 & $88,19 a$ & O & +1 \\
\hline 16 & $88,62 \mathrm{ab}$ & +1 & $67,22 \mathrm{ab}$ & $\mathrm{O}$ & 90,89 a & +2 & 88,29 a & $\mathrm{O}$ & +3 \\
\hline 17 & $87,64 \mathrm{ab}$ & +1 & $68,86 \mathrm{ab}$ & O & $89,90 \mathrm{ab}$ & +1 & $86,46 a$ & $\mathrm{O}$ & +2 \\
\hline 18 & $87,75 \mathrm{ab}$ & +1 & $66,03 \mathrm{ab}$ & O & $89,06 \mathrm{ab}$ & +1 & $89,45 \mathrm{a}$ & O & +2 \\
\hline 19 & $89,10 \mathrm{ab}$ & +1 & $72,61 \mathrm{ab}$ & O & $90,67 \mathrm{a}$ & +2 & 89,90 a & $\mathrm{O}$ & +3 \\
\hline 20 & $89,69 \mathrm{ab}$ & +1 & $71,63 \mathrm{ab}$ & O & $90,31 \mathrm{ab}$ & +1 & 89,35 a & O & +2 \\
\hline 21 & $87,02 \mathrm{ab}$ & +1 & $69,92 \mathrm{ab}$ & O & $90,64 \mathrm{a}$ & +2 & $87,28 \mathrm{a}$ & $\mathrm{O}$ & +3 \\
\hline 22 & $87,78 \mathrm{ab}$ & +1 & $70,68 \mathrm{ab}$ & $\mathrm{O}$ & $90,22 \mathrm{ab}$ & +1 & $86,45 \mathrm{a}$ & $\mathrm{O}$ & +2 \\
\hline 23 & $85,74 \mathrm{ab}$ & +1 & $69,52 \mathrm{ab}$ & O & $88,81 \mathrm{ab}$ & +1 & 87,08 a & $\mathrm{O}$ & +2 \\
\hline 24 & $87,55 \mathrm{ab}$ & +1 & $65,70 \mathrm{ab}$ & O & $90,32 \mathrm{ab}$ & +1 & $88,61 \mathrm{a}$ & O & +2 \\
\hline 25 & $87,76 \mathrm{ab}$ & +1 & $65,15 \mathrm{ab}$ & $\mathrm{O}$ & $90,74 \mathrm{a}$ & +2 & $87,73 \mathrm{a}$ & $\mathrm{O}$ & +3 \\
\hline 26 & $90,77 \mathrm{a}$ & +2 & $72,58 \mathrm{ab}$ & O & $89,20 \mathrm{ab}$ & +1 & 89,42 a & O & +3 \\
\hline 27 & $87,21 \mathrm{ab}$ & +1 & $68,05 \mathrm{ab}$ & O & $90,33 \mathrm{ab}$ & +1 & 87,49 a & $\mathrm{O}$ & +2 \\
\hline 28 & $85,66 \mathrm{ab}$ & +1 & $69,92 \mathrm{ab}$ & O & 91,04 a & +2 & 88,81 a & $\mathrm{O}$ & +3 \\
\hline
\end{tabular}

(1) Médias seguidas de mesma letra na coluna não diferem entre si pelo Teste de Tukey a $5 \%$ de probabilidade.

TABELA 6. Classificação estatística: distribuição dos lotes em grupos superior (SM), inferior (IM) e médio (M) nos conjuntos A (determinações de laboratório) e B (determinações de campo); taxa de coincidência (TC), entre os conjuntos A e B, calculada a partir do número de presenças no mesmo grupo.

\begin{tabular}{|c|c|c|c|c|c|}
\hline \multirow[b]{2}{*}{ Lote $\mathbf{n}^{\circ}$} & \multicolumn{2}{|c|}{ Conjunto A (laboratório) } & \multicolumn{2}{|c|}{ Conjunto B (campo) } & Conjuntos A e B \\
\hline & $\begin{array}{c}\text { Pontuação } \\
\text { geral }\end{array}$ & $\begin{array}{c}\text { Grupo } \\
\mathrm{M}=\mathrm{O}\end{array}$ & $\begin{array}{l}\text { Pontuação } \\
\text { geral }\end{array}$ & $\begin{array}{c}\text { Grupo } \\
\mathrm{M}=\mathrm{O}\end{array}$ & $\begin{array}{l}\text { Presenças no } \\
\text { mesmo grupo }\left(\mathbf{n}^{\circ}\right)\end{array}$ \\
\hline 1 & -31 & IM & +1 & SM & $\mathrm{O}$ \\
\hline 2 & +11 & SM & +1 & SM & 1 \\
\hline 3 & +1 & SM & O & $\mathbf{M}$ & O \\
\hline 4 & -80 & IM & +1 & SM & O \\
\hline 5 & -76 & IM & -40 & IM & 1 \\
\hline 6 & +2 & SM & O & $\mathbf{M}$ & $\mathrm{O}$ \\
\hline 7 & +38 & SM & +2 & SM & 1 \\
\hline 8 & -62 & IM & -6 & IM & 1 \\
\hline 9 & -12 & IM & +2 & SM & O \\
\hline 10 & +23 & SM & +1 & SM & 1 \\
\hline 11 & -24 & IM & +2 & SM & O \\
\hline 12 & -8 & IM & +2 & SM & O \\
\hline 13 & -1 & IM & O & $\mathbf{M}$ & $\mathrm{O}$ \\
\hline 14 & +19 & SM & +1 & SM & 1 \\
\hline 15 & -6 & IM & +1 & SM & O \\
\hline 16 & +20 & SM & +3 & SM & 1 \\
\hline 17 & +17 & SM & +2 & SM & 1 \\
\hline 18 & +35 & SM & +2 & SM & 1 \\
\hline 19 & +47 & SM & +3 & SM & 1 \\
\hline 20 & +19 & SM & +2 & SM & 1 \\
\hline 21 & +51 & SM & +3 & SM & 1 \\
\hline 22 & +1 & SM & +2 & SM & 1 \\
\hline 23 & +17 & SM & +2 & SM & 1 \\
\hline 24 & -13 & IM & +2 & SM & $\mathbf{O}$ \\
\hline 25 & -19 & IM & +3 & SM & O \\
\hline 26 & +40 & SM & +3 & SM & 1 \\
\hline 27 & +4 & SM & +2 & SM & 1 \\
\hline \multirow[t]{3}{*}{28} & +11 & SM & +3 & SM & 1 \\
\hline & & & & & $\begin{array}{ll}\text { Total } & 17 \\
\end{array}$ \\
\hline & & & & & $\mathrm{TC}=17.28^{-1} \cdot 100=60,71 \%$ \\
\hline
\end{tabular}




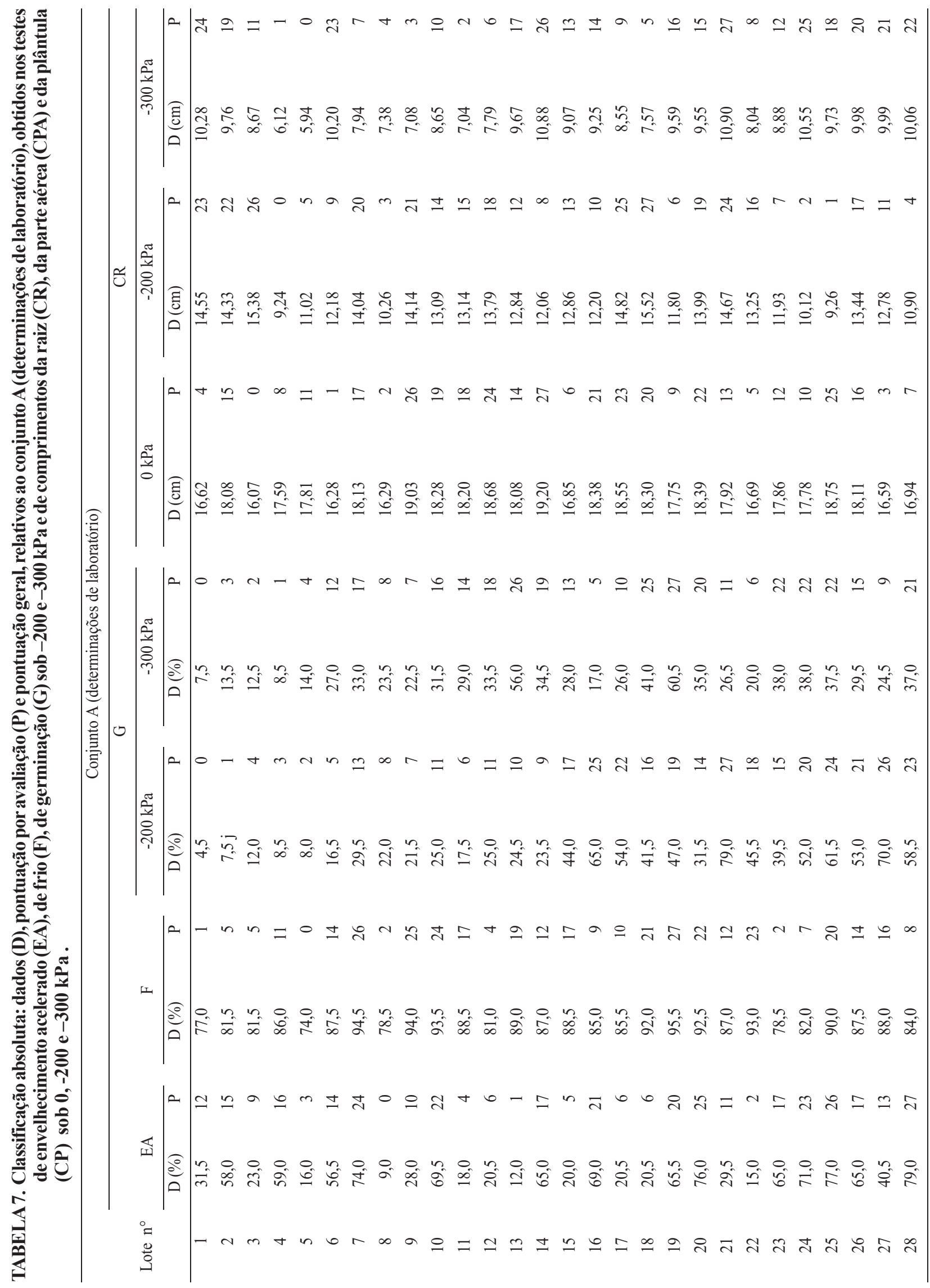




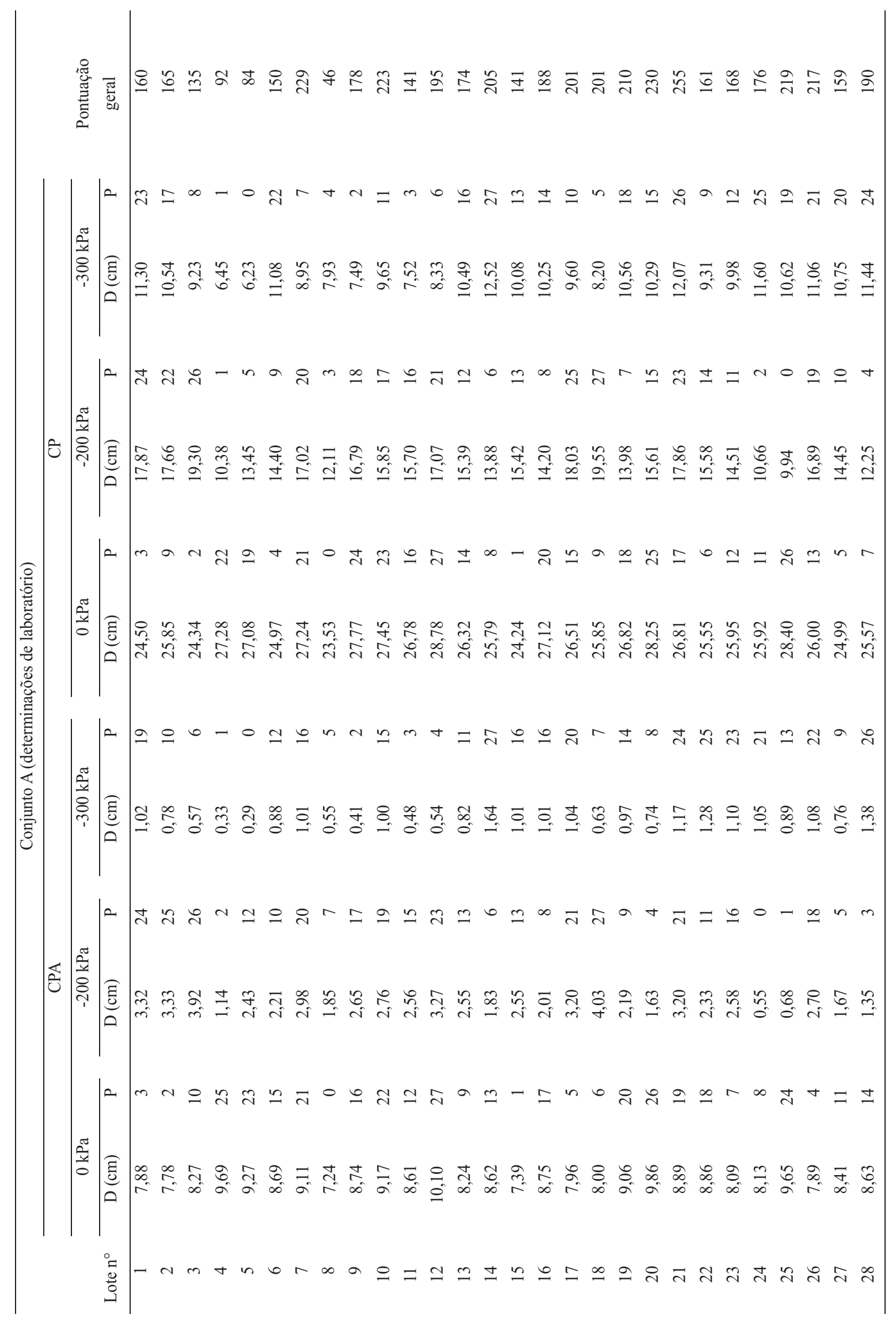


TABELA 8. Classificação absoluta: dados (D), pontuação por avaliação (P) e pontuação geral, relativos ao conjunto B (determinações de campo), obtidos nos testes de emergência das plântulas (EP).

\begin{tabular}{|c|c|c|c|c|c|c|c|c|c|}
\hline \multirow{3}{*}{ Lote $\mathrm{n}^{\circ}$} & \multicolumn{8}{|c|}{ Conjunto B (determinações de campo) } & \multirow{3}{*}{ Pontuação geral } \\
\hline & \multicolumn{2}{|c|}{ EP (época 1) } & \multicolumn{2}{|c|}{ EP (época 2) } & \multicolumn{2}{|c|}{ EP (época 3) } & \multicolumn{2}{|c|}{ EP (época 4) } & \\
\hline & $\mathrm{D}(\%)$ & $\mathrm{P}$ & $\mathrm{D}(\%)$ & $\mathrm{P}$ & $\mathrm{D}(\%)$ & $\mathrm{P}$ & $\mathrm{D}(\%)$ & $\mathrm{P}$ & \\
\hline 1 & 87,88 & 22 & 62,03 & 4 & 86,64 & 5 & 89,19 & 18 & 49 \\
\hline 2 & 86,17 & 11 & 63,10 & 6 & 85,83 & 4 & 89,94 & 25 & 46 \\
\hline 3 & 82,50 & 2 & 67,49 & 15 & 86,68 & 6 & 86,94 & 5 & 28 \\
\hline 4 & 87,80 & 19 & 61,77 & 3 & 85,37 & 3 & 86,75 & 4 & 29 \\
\hline 5 & 73,30 & 0 & 51,48 & 0 & 80,62 & 0 & 83,24 & 0 & 0 \\
\hline 6 & 82,56 & 3 & 61,60 & 2 & 87,46 & 8 & 87,97 & 12 & 25 \\
\hline 7 & 88,15 & 23 & 64,83 & 10 & 89,70 & 16 & 89,40 & 20 & 69 \\
\hline 8 & 80,94 & 1 & 64,06 & 9 & 83,20 & 1 & 83,92 & 1 & 12 \\
\hline 9 & 87,82 & 20 & 56,51 & 1 & 89,15 & 14 & 87,78 & 11 & 46 \\
\hline 10 & 85,97 & 10 & 69,57 & 19 & 88,35 & 10 & 89,50 & 23 & 62 \\
\hline 11 & 87,84 & 21 & 70,22 & 22 & 89,82 & 18 & 90,48 & 27 & 88 \\
\hline 12 & 83,82 & 6 & 73,28 & 27 & 88,06 & 9 & 90,22 & 26 & 68 \\
\hline 13 & 83,82 & 5 & 63,33 & 7 & 84,57 & 2 & 89,15 & 17 & 31 \\
\hline 14 & 85,62 & 7 & 62,32 & 5 & 86,78 & 7 & 87,54 & 9 & 28 \\
\hline 15 & 86,58 & 4 & 63,43 & 8 & 88,80 & 11 & 88,19 & 13 & 36 \\
\hline 16 & 88,62 & 24 & 67,22 & 14 & 90,89 & 26 & 88,29 & 14 & 78 \\
\hline 17 & 87,64 & 15 & 68,86 & 17 & 89,90 & 17 & 86,46 & 3 & 52 \\
\hline 18 & 87,75 & 16 & 66,03 & 13 & 89,06 & 13 & 89,45 & 22 & 64 \\
\hline 19 & 89,10 & 25 & 72,61 & 26 & 90,67 & 24 & 89,90 & 24 & 99 \\
\hline 20 & 89,69 & 26 & 71,63 & 24 & 90,31 & 20 & 89,35 & 19 & 89 \\
\hline 21 & 87,02 & 12 & 69,92 & 20 & 90,64 & 23 & 87,28 & 7 & 62 \\
\hline 22 & 87,78 & 18 & 70,68 & 23 & 90,22 & 19 & 86,45 & 2 & 62 \\
\hline 23 & 85,74 & 9 & 69,52 & 18 & 88,81 & 12 & 87,08 & 6 & 45 \\
\hline 24 & 87,55 & 14 & 65,70 & 12 & 90,32 & 21 & 88,61 & 15 & 62 \\
\hline 25 & 87,76 & 17 & 65,15 & 11 & 90,74 & 25 & 87,73 & 10 & 63 \\
\hline 26 & 90,77 & 27 & 72,58 & 25 & 89,20 & 15 & 89,42 & 21 & 88 \\
\hline 27 & 87,21 & 13 & 68,05 & 16 & 90,33 & 22 & 87,49 & 8 & 59 \\
\hline 28 & 85,66 & 8 & 69,92 & 21 & 91,04 & 27 & 88,81 & 16 & 72 \\
\hline
\end{tabular}

Assim, diante da dificuldade de interpretar dados de vigor oriundos de distintas determinações, a adoção de critérios de pontuação pode constituir alternativa para hierarquizar lotes segundo o desempenho geral e, paralelamente, para inter- relacionar as respostas de testes realizados em laboratório e em campo. Levando em conta as opções estudadas, a classificação absoluta apresenta-se mais vantajosa do que a classificação estatística para essas finalidades. 
TABELA 9. Classificação absoluta: distribuição dos lotes em grupos superior (SM), inferior (IM) e médio (M) nos conjuntos A (determinações de laboratório) e B (determinações de campo); taxa de coincidência (TC), entre os conjuntos A e B, calculada a partir do número de presenças no mesmo grupo.

\begin{tabular}{|c|c|c|c|c|c|}
\hline \multirow[b]{2}{*}{ Lote $n^{\circ}$} & \multicolumn{2}{|c|}{ Conjunto A (laboratório) } & \multicolumn{2}{|c|}{ Conjunto B (campo) } & \multirow{2}{*}{$\begin{array}{c}\text { Conjuntos A e B } \\
\text { Presenças no } \\
\text { mesmo grupo }\left(\mathrm{n}^{\circ}\right)\end{array}$} \\
\hline & $\begin{array}{c}\text { Pontuação } \\
\text { geral }\end{array}$ & $\begin{array}{c}\text { Grupo } \\
\mathrm{M}=(255+46) \cdot 2^{-1}=150,5\end{array}$ & $\begin{array}{c}\text { Pontuação } \\
\text { geral }\end{array}$ & $\begin{array}{c}\text { Grupo } \\
\mathrm{M}=(99+0) \cdot 2^{-1}=49,5\end{array}$ & \\
\hline 1 & 160 & SM & 49 & IM & 0 \\
\hline 2 & 165 & $\mathrm{SM}$ & 46 & IM & 0 \\
\hline 3 & 135 & IM & 28 & IM & 1 \\
\hline 4 & 92 & IM & 29 & IM & 1 \\
\hline 5 & 84 & IM & 0 & IM & 1 \\
\hline 6 & 150 & IM & 25 & IM & 1 \\
\hline 7 & 229 & SM & 69 & SM & 1 \\
\hline 8 & 46 & IM & 12 & IM & 1 \\
\hline 9 & 178 & SM & 46 & IM & 0 \\
\hline 10 & 223 & SM & 62 & SM & 1 \\
\hline 11 & 141 & IM & 88 & SM & 0 \\
\hline 12 & 195 & SM & 68 & SM & 1 \\
\hline 13 & 174 & SM & 31 & IM & 0 \\
\hline 14 & 205 & $\mathrm{SM}$ & 28 & IM & 0 \\
\hline 15 & 141 & IM & 36 & IM & 1 \\
\hline 16 & 188 & SM & 78 & SM & 1 \\
\hline 17 & 201 & SM & 52 & SM & 1 \\
\hline 18 & 201 & SM & 64 & $\mathrm{SM}$ & 1 \\
\hline 19 & 210 & SM & 99 & SM & 1 \\
\hline 20 & 230 & $\mathrm{SM}$ & 89 & $\mathrm{SM}$ & 1 \\
\hline 21 & 255 & SM & 62 & SM & 1 \\
\hline 22 & 161 & SM & 62 & SM & 1 \\
\hline 23 & 168 & SM & 45 & IM & 0 \\
\hline 24 & 176 & SM & 62 & $\mathrm{SM}$ & 1 \\
\hline 25 & 219 & SM & 63 & SM & 1 \\
\hline 26 & 217 & SM & 88 & SM & 1 \\
\hline 27 & 159 & SM & 59 & SM & 1 \\
\hline 28 & 190 & SM & 72 & SM & 1 \\
\hline & & & & & Total $\quad 21$ \\
\hline & & & & & $\mathrm{TC}=21.28^{-1} \cdot 100=75 \%$ \\
\hline
\end{tabular}

\section{CONCLUSÃO}

A interpretação de dados de vigor, oriundos de um conjunto de testes, adquire complexidade diretamente proporcional aos números de testes e de lotes colocados em comparação. Nessa situação, a classificação absoluta supera a classificação estatística nas tarefas de hierarquizar qualitativamente lotes de sementes de milho, segundo o desempenho geral, e de quantificar o relacionamento entre as respostas provenientes dos testes de laboratório com as obtidas em campo.

\section{AGRADECIMENTOS}

Os autores agradecem Antonio Dominciano Poletti, Cláudio Hagime Funai, José Orilton Franco Pereira, José Alexandre Contente e Roberto Annunciato Junior pela condução dos testes de emergência no campo.

\section{REFERÊNCIAS}

ASSOCIATION OF OFFICIAL SEED ANALYSTS. Seed vigour testing handbook. East Lasing: AOSA, 1983. 88p. Contribution, 32. 
BRASIL. Ministério da Agricultura e da Reforma Agrária. Regras para análise de sementes. Brasília: SNDA/DNDV/CLAV, 1992. $365 \mathrm{p}$.

CALIARI, M.F.; SILVA, W.R. Interpretação de dados de testes de vigor na avaliação da qualidade fisiológica de sementes de milho. Revista Brasileira de Sementes, Campinas, v.23, n.1, p.239-251, 2001.

CARVAlho, N.M.; NAKAGAWA, J. Sementes: ciência, tecnologia e produção. Jaboticabal: FUNEP, 2000, 588p.

GRABE, D.F. Measurement of seed vigor. Journal of Seed Technology, Lansing, v.1, n.2, p.18-31, 1976.

HAMPTON, J.C.; COOLBEAR, P. Potential versus actual seed performance - can vigor testing provide an answer? Seed Science and Technology, Zürich, v.18, n.2, p.215-228, 1990.

INTERNACIONAL SEED TESTING ASSOCIATION. Handbook of vigour test methods. Zürich: ISTA, 1981. 72p.
MARCOS FILHO, J.; PESCARIN, H.M.C.; KOMATSU, Y.H.; DEMÉTRIO, C.G.B.; FANCELLI, A.L. Testes para avaliação do vigor de sementes de soja e sua relação com a emergência das plântulas em campo. Pesquisa Agropecuária Brasileira, Brasília, v.19, n.5, p.605-613, 1984.

MARCOS FILHO, J.; CICERO, S.M.; SILVA, W.R. Avaliação da qualidade das sementes. Piracicaba: FEALQ, 1987. 230p.

MARCOS FILHO, J. Importância dos testes de vigor. Seed News, Pelotas, Edição n.6 (julho/agosto), p.32, 1998.

VIEIRA, R.D.; CARVALHO, N.M.; SADER, R. Testes de vigor e suas possibilidades de uso. In: VIEIRA, R.D.; CARVALHO, N.M. (Ed.) Testes de vigor em sementes. Jaboticabal: FUNEP, 1994.164p.

VILLELA, F.A.; DONI FILHO, L.; SEQUEIRA, E.L. Tabela de potencial osmótico em função da concentração de polietileno glicol 6000 e da temperatura. Pesquisa Agropecuária Brasileira, Brasilia, v.26, n. 11/12, p. 1957-1968, 1991. 